\title{
ELECTRONIC SPACES AND CLASSIFICATION OF MULTIELECTRON STATES IN MULTICENTRE SYSTEMS
}

\author{
R. Chatterjee ${ }^{a}$, T. Lulek ${ }^{b}$ and J.K. Biel ${ }^{a}$ \\ a Department of Physics, University of Calgary, Calgary, Alberta T2N 1N4, Canada \\ ${ }^{6}$ Institute of Physics, A. Mickiewicz University, Matejki 48/49, 60-769 Poznań, Poland
}

(Received February 24, 1992)

\begin{abstract}
Electronic structure of a crystal within the tight binding model is described in terms of fibre bundle formalism, with the base and fiber being respectively the set of all sites and the single-centre space of electron spinorbitals. It is based on the Weyl's duality between the symmetric group and the unitary group, and paves the way for a Racah-Wigner type of description of electronic structure in multicentre systems.
\end{abstract}

PACS numbers: 02.40.+m, 33.10.Cs, 71.10.+x, 74.60.-w

\section{Introduction}

The aim of the present paper is to propose a concise fibre bundle description of electronic structure of multicentre systems (molecules, crystals, Bravais cells, etc.) within the tight binding [1] and itinerant electron [2] models, in a way adapted to the Wigner-Racah approach [3-7]. In these models one starts with two ingredients: (i) the set $I$ of all nodes of the system, each node $i \in I$ serving as a centre of localization of electrons, (ii) a finite-dimensional single-centre unitary space $W$ of electron spinorbitals. Mathematically, it has been proved convenient [3] to look at these two ingredients as the building elements of a bundle $[8,9]$ with the discrete base $I$ and the typical fiber $W$. The associated factorization of the space $L$ of all multicentre spinorbitals (i.e. molecular spinorbitals of the MO LCAO method, or Bloch functions of the tight binding approach) into positional and polarizational parts [3-7] provides the ground for a construction of multielectron multicentre bases by means of an appropriate multiple coupling within the Racah-Wigner scheme. This approach has been already applied to determination of two-electron states in molecules [4] and, in particular, to Cooper-like pairs on a square [5]. Here we extend this method to the case of an arbitrary number $N$ of electrons, replacing a detailed multiple coupling of single-electron spaces by a scheme suggested by the 
general Weyl's duality between the actions of the symmetric group $\Sigma_{N}$ and some unitary groups in the relevant tensor spaces.

\section{Types of electronic spaces}

We start our description of electronic structure of multicentre systems within the tight binding approach with a careful distinction of a number of relevant quantum Hilbert spaces. On the one hand, we have to distinguish between single-centre and multicentre, or crystalline states, and on the other hand between single-electron and multielectron ones. Thus $W$ and $L$ are single-electron spaces for the singleand multicentre cases, respectively, so that the electron Bloch states are vectors of $L$, whereas, e.g. the hydrogenic $1 s$ state belongs to $W$. We assume that $W$ is truncated to a finite-dimensional space of some optimised spinorbitals. Thus $n=\operatorname{dim} W=2,6,10$ and 14 for $s, p, d$, and $f$ electrons, respectively.

Within the tight binding approach, the single-electron multicentre space $L$ is spanned on the set $I$ of all centres, so that $\operatorname{dim} L=|I| \operatorname{dim} W$. The elements of $L$ are thus extended states of a single electron. Such states involve both atomic character of localised orbitals via the space $W$ and the geometric symmetry of a multicentre system, expressed by covariant bases, adapted to the structure of the set $I$ (e.g. Bloch functions for a periodic structure on $I$ ).

According to the Pauli exclusion principle, the $N$-electron spaces are antisymmetrised $N$-th tensor powers of corresponding single-electron spaces, i.e. $W^{\left\{1^{N}\right\}}$ and $L^{\left\{1^{N}\right\}}$ for the atomic and multicentre case, respectively, with $\left\{1^{N}\right\}$ denoting the Young diagram for the partition of the integer $N$ into $N$ parts. Thus $W^{\left\{1^{\circ}\right\}}$ and $L^{\left\{1^{\circ}\right\}}$ are one-dimensional vacuum spaces, $W^{\left\{1^{1}\right\}}=W$ and $L^{\left\{1^{1}\right\}}=L$ correspond to a single electron, $W^{\left\{1^{2}\right\}}$ is the space of all atomic pairs of electrons, $L^{\left\{1^{2}\right\}}$ involves extended Cooper pairs, etc. Then the Fock spaces, appropriate for systems with undefinite number of electrons, are

$$
F=\sum_{N=0}^{\operatorname{dim} W} \oplus W^{\left\{1^{N}\right\}}
$$

and

$$
Y=\sum_{N=1}^{\operatorname{dim} L} \oplus L^{\left\{1^{N}\right\}}
$$

for the atomic and multicentre case, respectively.

The single-centre Fock space $F$ provides the starting point for the atomic representation in the Hubbard model of itinerant electrons [2]. If, e.g. the single-centre space $W$ is spanned over all $n l$ spinorbitals for fixed $n l$ atomic quantum numbers, then the Fock space $F$ is spanned over all states of atomic shells $(n l)^{N}$ for $N=0,1,2, \ldots, 2(2 l+1)$. In particular, in the case of $s$-electrons we have only spin degrees of freedom, so that $\operatorname{dim} W=2$, and

$$
F=W^{\left\{1^{\circ}\right\}} \oplus W^{\left\{1^{1}\right\}} \oplus W^{\left\{1^{2}\right\}},
$$

with the consecutive subspaces $W^{\left\{1^{N}\right\}}$ spanned over the empty-site state $\{-\}$, singly occupied spin states $\{\uparrow, \downarrow\}$, and the doubly occupied antisymmetric state $\{\uparrow \downarrow\}$, so that $\operatorname{dim} F=1+2+1=4$. 
The crystalline Fock space $Y$ is the principal object for a quantum description of electron phenomena in molecules and crystals within the tight binding model. When the single-centre space $W$ is of a finite dimension, and the set $I$ of sites is finite, then the Fock space $Y$ is also finite-dimensional, namely

$$
\operatorname{dim} Y=2^{|\Gamma| \operatorname{dim} W} .
$$

\section{Fibre bundle structure of electronic spaces}

Within the tight binding approach, the principal model assumption is the choice of the single-centre, single-electron space $W$, whereas all the other spaces, i.e. $F, L$, and $Y$, have to be constructed using the known distribution of sites in crystals. The latter is coded as a structure of the geometric symmetry grup $G$, imposed on the set $I$ of all sites. Construction of the single-centre Fock space $F$ consists merely in multiple coupling of $N$ copies of the single-electron space $W$ using the Pauli exclusion principle [10], within the Wigner-Racah approach [11]. On the other hand, construction of multicentre spaces $L$ and $Y$ involves, in an intrinsic way, an approach based on fibre bundle techniques [3-9]. We proceed to describe this approach briefly for the case of the multicentre single-electron space $L$.

Let $\phi_{i}: W \rightarrow W_{i}$ be the isomorphism of spaces which produces a faithful copy $W_{i}$ of the unitary space $W$, centred at the site $i \in I$. The set of all such copies, i.e.

$$
E=\bigcup_{i \in I} W_{i},
$$

is the fibre bundle with the discrete base $I$ and the typical fiber $W$. The elements of the bundle $E$ are thus single-electron states $e \in W_{i} \subset E$, each localized at a definite site $i \in I$. The identification of a node is provided by the canonical projection $p: E \rightarrow I$ of the bundle $E$ onto its base $I$, defined by $p(e)=i$ for $e \in W_{i} \subset E$. The section of the bundle $E$ is defined as any mapping $\psi: I \rightarrow E$ satisfying $p \circ \psi=i d_{I}$, where $i d_{I}: I \rightarrow I$ denotes the identity mapping on $I$. Now, the space $L$ of single-electron multicentre states is identified with the set of all sections $\psi$ of the bundle $E$. The sum $\psi_{1}+\psi_{2}$ and multiplication by complex numbers $c \psi$ is defined pointwise, i.e. $\left(\psi_{1}+\psi_{2}\right)(i)=\psi_{1}(i)+\psi_{2}(i), i \in I$, with the sign " + " in the left- and right-hand side being the addition in $L$ and $W_{i}$, respectively, and $(c \psi)(i)=c \psi(i), i \in I$.

In order to adapt the above "coordinate free" description to some reference frames, we introduce an orthonormal basis $\tilde{n}=\{\alpha=1,2, \ldots, n\}$ in the typical fiber $W$. Then the set $\left\{\phi_{i}(\alpha) \mid \alpha \in \tilde{n}\right\}$ is an orthonormal basis in the space $W_{i}$ of the fiber over the site $i \in I$, and the formula

$$
\psi_{i \alpha}\left(i^{\prime}\right)=\psi_{i}(\alpha) \delta_{i i^{\prime}}, \quad i^{\prime} \in I,
$$

defines the section $\psi_{i \alpha} \in L$. The set

$$
\varepsilon=\left\{\psi_{i \alpha} \equiv|i, \alpha>| i \in I, \alpha \in \tilde{n}\right\} \cong I \times \tilde{n}
$$

is a basis in the linear space $L$ of all sections of the bundle $E$. The unitary structure of $L$ is imposed by the requirement that $\varepsilon$ is an orthonormal basis, i.e. that

$$
\left\langle i ; \alpha \mid i^{\prime} ; \alpha^{\prime}\right\rangle=\delta_{i i^{\prime}} \delta_{\alpha \alpha^{\prime}}, \quad i, i^{\prime} \in I, \quad \alpha, \alpha^{\prime} \in \tilde{n} .
$$


It corresponds to neglecting any overlap of electrons localised at different centres. Within this description, an arbitrary single-electron multicentre state is characterised by the unique expansion

$$
\left|\psi>=\Sigma_{i \in I} \Sigma_{\alpha \in \tilde{n}} c_{i \alpha}\right| i ; \alpha>
$$

with some complex coefficients $c_{i \alpha}$. Briefly, a molecular spinorbital $|\psi\rangle$ of the famous MO LCAO method is a section of the bundle $E$.

It is worth to observe that the structure of our bundle $E$ is much simplified in comparison to bundles considered in textbooks of differential geometry [8,9]. Namely, our base $I$ is a discrete set, which can even be made finite by means of Born-Kárman periodic conditions, whereas the textbook base is usually equipped with the structure of a differential manifold. Thus the so-called "triviality condition" imposing the local Cartesian product. structure to each open neighbourhood in the base $I$ becomes redundant, and the set of mappings $\left\{\phi_{i} \mid i \in I\right\}$ provides a global structure for the whole bundle $E$. Still, even then there is an essential difference between the Cartesian product $I \times W$ and the bundle $E$ since the former has two canonical projections $\pi_{1}: I \times W \rightarrow I$ and $\pi_{2}: I \times W \rightarrow W$ onto each factor, whereas the latter disposes only one canonical projection $p: E \rightarrow I$ onto the base [9]. Mappings $\phi_{i}^{-1}: W_{i} \rightarrow W$ do exist, but are not canonical, since the basis on each fiber $W_{i}$ can be chosen independently. In short, the base $I$ is "absolute", whereas the fiber $W$ is "relative" $[3,12]$. Such a presentation of discrete structures in terms of bundles is similar to Mozrzymas [13] description of space groups as bundles with the point and translation groups playing respectively the role of the base and typical fiber.

\section{Factorization, the duality of Weyl, and Wigner-Racah type of approach}

Fibre structure of the space $L$ of multicentre single-electron states, presented in the preceding Section, in particular Eqs. (5) and (7), suggests a factorization

$$
L=B \otimes W,
$$

where

$$
B=l c_{C} I
$$

is the linear space, constructed as the formal linear closure $(l c)$ of the set $I$ of sites, over the field $C$ of complex numbers. The unitary structure of $B$ is imposed by requiring $I$ to be an orthonormal basis. We refer hereafter to $B$ as the positional space [14-16]. Equation (10) provides thus a factorization of the single-electron space $L$ into the positional factor $B$, accounting for the geometric distribution of centres, and the polarizational factor $W$, related to the single-centre spinorbitals.

The factorization (10) bears a far-reaching resemblance to atomic case, where spin variables are coupled to orbital ones. This fact paves the way for a Wigner-Racah type of approach in constructing some convenient bases in multielectron spaces. We consider for illustration the case $N=2$ [4-7]. The obvious isomorphism

$$
L^{2}=(B \otimes W) \otimes(B \otimes W) \cong(B \otimes B) \otimes(W \otimes W)
$$


yields various Wigner-Racah types of coupling schemes, recoupling coefficients, etc., in a full analogy to addition of four angular momenta in terms of $9 j$-symbols. The symmetrization can be thus performed separately for each of both factors, positional $B \otimes B$ and polarizational $W \otimes W$. In particular, the space of two-electron states can be witten as

$$
L^{\left\{1^{2}\right\}} \cong\left(B^{\{2\}} \otimes W^{\left\{1^{2}\right\}}\right) \oplus\left(B^{\left\{1^{2}\right\}} \otimes W^{\{2\}}\right) .
$$

We obtain thus two subspaces, each being a direct product of the positional and polarizational factor. Each factor is bilinear in electron indices and exhibits a definite statistics, $\{2\}$ or $\left\{1^{2}\right\}$, so that the resultant Young diagram is antisymmetric to fulfil the Pauli exclusion principle. For finite sets $I$ and $\tilde{n}$, the counting formula for dimensions in Eq. (13) reads

$$
\left(\begin{array}{c}
|I| n \\
2
\end{array}\right)=\frac{|I|(|I|+1)}{2}\left(\begin{array}{c}
n \\
2
\end{array}\right)+\left(\begin{array}{c}
|I| \\
2
\end{array}\right) \frac{n(n+1)}{2}
$$

This discussion can be extended to an arbitrary number $N$ of electrons by use of appropriate coupling schemes. We are not going here to describe any detailed scheme of a multiple coupling, but only sketch out the way of classification of basic states in terms of the Weyl's duality $[17,18]$ between the actions of the symmetric group $\Sigma_{N}$ of permutations of electrons, referred to as the Pauli group, and of the single-particle unitary group, $U(n)$ or $U(n|I|)$, in appropriate tensor power spaces.

We start with the formulation of the duality of Weyl for the case of the $N$-th tensor power space $W^{N}$ of the single-centre space $W$. Let $P_{W}: \Sigma_{N} \times W^{N} \rightarrow W^{N}$ be the action of the Pauli group $\Sigma_{N}$ in $W^{N}$, and let $V_{W}^{N}: U(n) \times W^{N} \rightarrow W^{N}$ be the action of the unitary group $U(n)$ in $W^{N}$. Thus $P_{W}(\sigma), \sigma \in \Sigma_{N}$, permutes electrons between various factors $W$ of $W^{N}$ and $V_{W}^{N}(g), g \in U(n)$, performs the same unitary transformation $g$ in each factor. These two actions commute mutually, i.e.

$$
P_{W}(\sigma) V_{W}^{N}(g)=V_{W}^{N}(g) P_{W}(\sigma), \quad \sigma \in \Sigma_{N}, \quad g \in U(n) .
$$

Thus the basis states in the space $W^{N}$ can be classified by irreducible representations $\Delta^{\Lambda}$ of the Pauli group $\Sigma_{N}$ and $D^{\Lambda}$ of the unitary group $U(n)$ simultaneously, both irreducible representations being labelled by the same Young diagram $\Lambda$, corresponding to a partition of $N$ into $n$ parts. The Weyl's duality states that the multiplicity $m\left(P_{W}, \Delta^{\Lambda}\right)$ of $\Delta^{\Lambda}$ in $P_{W}$ is equal to the dimension of $D^{\Lambda}$ and, vice versa, the multiplicity $m\left(V_{W}^{N}, D^{\Lambda}\right)$ of $D^{\Lambda}$ in $V_{W}^{N}$ is equal to the dimension of $\Delta^{\Lambda}$. Moreover, these multiplicities are given by hooklength formulas, so that

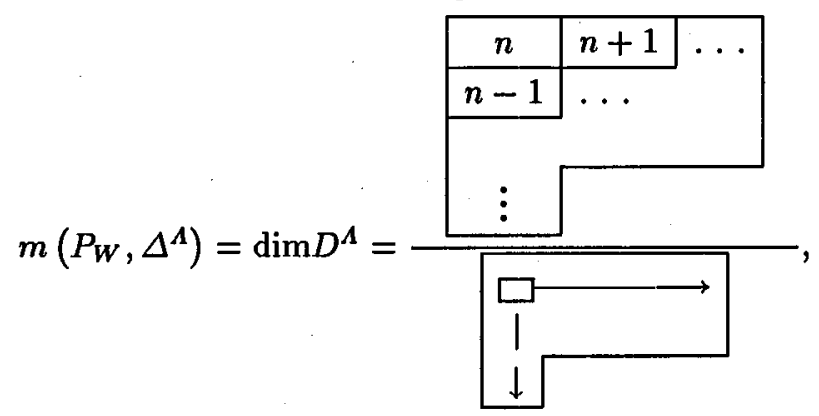


and

$$
m\left(V^{N}, D^{\Lambda}\right)=\operatorname{dim} \Delta^{\Lambda}=\frac{N !}{\square}
$$

where contours of the Young diagram $\Lambda$ denote products of all its entries, and each entry in denominators is the appropriate hooklength - the number of boxes encompassed by the hook formed by indicated arrows. Thus the entry of the $a$-th row (of length $\lambda_{a}$ ) and the $b$-th column (of the length $\lambda_{b}^{\prime}$ ) is $\lambda_{a}+\lambda_{b}^{\prime}-a-b+1$.

Thus the actions of groups of the duality of Weyl, $\Sigma_{N}$ and $U(n)$, in the space $W^{N}$ yields the decomposition

$$
W^{N}=\sum_{\Lambda} \oplus W^{\Lambda}
$$

into subspaces $W^{\Lambda}$, invariant under both groups. The restriction $\left.P_{W}\right|_{W^{\Lambda}}$ of the action $P_{W}$ of $\Sigma_{N}$ to the subspace $W^{A}$ consists of $m\left(P_{W}, \Delta^{A}\right)$ copies of the irreducible representation $\Delta^{\Lambda}$ of $\Sigma_{N}$, and the restriction $\left.V_{W}^{N}\right|_{W^{\Lambda}}$ of the action $V_{W}^{N}$ to this subspace is the $m\left(V_{W}^{N}, D^{\Lambda}\right)$-fold repetition of the irreducible representation $D^{\Lambda}$ of $U(n)$. It is important to point out that both irreducible representations, $\Delta^{\Lambda}$ and $D^{\Lambda}$, are labelled by the same Young diagram $\Lambda$. The sum rule corresponding to the Weyl's duality (16)-(17) reads

$$
\sum_{\Lambda} m\left(P_{W}, \Delta^{\Lambda}\right) m\left(V_{W}^{N}, D^{\Lambda}\right)=n^{N}
$$

where the sum runs over all non-increasing partitions $A$ of $N$ into not more than $n$ parts $\lambda_{i}, i \in \tilde{n}$, ordered such that $0<\lambda_{i+1} \leq \lambda_{i}, \Sigma_{i \in \tilde{n}} \lambda_{i}=N$.

Now we observe that the factorization (10) yields the isomorphism

$$
L^{N} \cong B^{N} \otimes W^{N}
$$

and that the duality of Weyl (16)-(17) can be applied to each factor, positional $B^{N}$ and polarizational $W^{N}$, separately. Then the antisymmetric subspace $L^{\left\{1^{N}\right\}}$ of $L^{N}$ can be constructed using the Clebsch-Gordan selection rule for the Pauli group $\Sigma_{N}$, given by

$$
m\left(\Delta^{\Lambda} \otimes \Delta^{\Lambda^{\prime}}, \Delta^{\left\{1^{N}\right\}}\right)= \begin{cases}1 & \text { if } \Lambda^{\prime}=\Lambda^{\operatorname{Tr}}, \\ 0 & \text { otherwise, }\end{cases}
$$

where $\Lambda^{\operatorname{Tr}}$ denotes the Young diagram transposed to $\Lambda$. Thus

$$
L^{\left\{1^{N}\right\}}=\sum_{\Lambda} \oplus\left(B^{\Lambda^{\mathrm{Tr}}}, W^{\Lambda}\right),
$$

where $\left(B^{\Lambda^{\mathrm{Tr}}}, W^{\Lambda}\right)$ denotes the antisymmetric part of the tensor product $B^{\Lambda^{\mathrm{Tr}}} \otimes$ $W^{\Lambda}$. Equation (22) is the generalization of Eq. (13) for an arbitary $N \leq n|I|$. The counting formula for dimensions reads now

$$
\left(\begin{array}{c}
n|I| \\
N
\end{array}\right)=\sum_{\Lambda} m\left(P_{B}, \Delta^{\Lambda^{\mathbf{T r}}}\right) m\left(P_{W}, \Delta^{\Lambda}\right),
$$


where appropriate multiplicities are given by the hooklength formula (16), with $n$ replaced by $|I|$ for the action $P_{B}: \Sigma_{N} \times B^{N} \rightarrow B^{N}$ of the Pauli group $\Sigma_{N}$ in the positional tensor power space $B^{N}$.

\section{An example}

We illustrate some features of our approach on an example of a simplified, finite two-dimensional crystal consisting of just four atoms, placed at corners of a square. It corresponds to cyclic boundary conditions with the Born-Kármán period $p=2$. We admit only localised $s$ electrons, so the single-centre Fock space $F$ is given by Eq. (3), and the crystalline Fock space $Y$ consists of subspaces $L^{\left\{1^{N}\right\}}$, $N=0,1,2, \ldots, 8$, with the dimensions

$$
\operatorname{dim} L^{\left\{1^{N}\right\}}=\operatorname{dim} L^{\left\{1^{8-N}\right\}}=\left(\begin{array}{c}
8 \\
N
\end{array}\right),
$$

and the sum rule

$$
2(1+8+28+56)+70=256 .
$$

The factor 2 in Eq. (25) accounts for the particle-hole symmetry of spaces $L\left\{1^{N}\right\}$ and $L^{\left\{1^{8-N}\right\}}$ for $N=0,1,2,3$, and the last term 70 corresponds to the half-filled case $N=4$.

The single-particle crystalline space $L$ is factorized according to Eq. (10) into the four-dimensional positional space $B$, spanned on indices of corners of the square, and two-dimensional spin space $W$. The one-dimensional spaces $L\left\{1^{\circ}\right\}$ and ${ }_{L}\left\{1^{8}\right\}$ correspond respectively to the vacuum state and to the full occupation of the crystal by electrons, the eight-dimensional spaces $L\left\{1^{1}\right\} \equiv L$ and $L^{\left\{1^{7}\right\} \cong L}$ describe respectively a single electron and a single hole and have the structure (10) of the direct product of the positional and spin part. The two-electron $L\left\{1^{2}\right\}$ and two-hole $L\left\{1^{6}\right\}$ spaces have the structure given by Eq. (13), i.e. they separate into the terms $B^{\{2\}} \otimes W^{\left\{1^{2}\right\}}$ (singlet pairing) and $B^{\left\{1^{2}\right\}} \otimes W^{\{2\}}$ (triplet pairing), each term having the direct product structure. The dimensional check reads

$$
6 \cdot 3+10 \cdot 1=28
$$

with $\Lambda=\{2\}$ and $\left\{1^{2}\right\}$ for the first and second term, respectively (cf. Eq. (12)).

The three-electron and three-hole spaces $L\left\{1^{3}\right\}$ and $L\left\{1^{5}\right\}$ have a more complicated structure, which is given according to Eq. (17) by

$$
L^{\left\{1^{3}\right\}}=\left(B^{\{3\}}, W^{\left\{1^{3}\right\}}\right) \oplus\left(B^{\{21\}}, W^{\{21\}}\right) .
$$

The first term corresponds to $S=3 / 2$, and the second to $S=1 / 2$. They are no more direct product spaces, but have a non-trivial structure resulting from separation of the antisymmetric part $L^{\left\{1^{3}\right\}}$ of $L^{3}$. For example, the positional part of the second term is neither purely fermionic, nor bosonic, but has a "parastatistic" $\Lambda=\{21\}$, corresponding to the two-dimensional irreducible representation of the Pauli group $\Sigma_{3}$. The counting of dimensions for Eq. (28) is

$$
56=4 \cdot 4+20 \cdot 2 \text {. }
$$


The space

$$
L^{\left\{1^{4}\right\}}=\left(B^{\left\{1^{4}\right\}}, W^{\{4\}}\right) \oplus\left(B^{\left\{21^{2}\right\}}, W^{\{31\}}\right) \oplus\left(B^{\left\{2^{2}\right\}}, W^{\left\{2^{2}\right\}}\right)
$$

corresponds to the half-filled square, and the consecutive terms correspond to the total spin $S=2,1$, and 0 . The dimensional check is

$$
70=1 \cdot 5+15 \cdot 3+20 \cdot 1 \text {. }
$$

\section{Final remarks and conclusions}

We have presented a description of electronic structure of a crystal within the tight binding approach in the language of fiber bundles. The base and typical fibre of this bundle is, respectively, the set $I$ of all lattice sites and the single-centre space $W$ of states of a localized electron. The factorization resulted from the fibre structure of multielectron spaces allows us to distinguish between positional and polarizational degrees of freedom. Our description is based on the Weyl's duality between the symmetric group $S_{N}$ and the unitary group $U(n), n=\operatorname{dim} W$, which yields the classification of irreducible subspaces by Young diagrams.

\section{References}

[1] H. Jones, The Theory of Brillouin Zones and Electronic States in Crystals, North-Holland, Amsterdam 1960.

[2] J.Hubbard, Proc. R. Soc. Lond. A, Math. Phys. Sci. 276, 238 (1963); 277, 2372 (1963); 281, 401 (1964); 285, 524 (1965).

[3] J.K. Biel, R. Chatterjee, T. Lulek, J. Chem. Phys. 86, 4531 (1987).

[4] T. Lulek, Acta Phys. Pol. A75, 487 (1989).

[5] J. Kawacki, K. Kłos, T. Lulek, Acta Phys. Pol. A78, 659 (1990).

[6].T. Lulek, M. Szopa, J. Phys. A, Math. Gen. 23, 677 (1990).

[7] T. Lulek, in: Symmetry and Structural Properties of Condensed Matter, Eds. W. Florek, T. Lulek, M. Mucha, World Scientific, Singapore 1991, p. 278.

[8] N. Steenrod, The Topology of Fibre Bundles, Princeton, New Jersey 1951.

[9] L. Auslander, R.E. MacKenzie, Introduction to Differentiable Manifolds, Mc Graw-Hill, New York 1963.

[10] W. Pauli, Z. Phys. 31, 735 (1925).

[11] L.C. Biedenharn, J.D. Louck, The Racah-Wigner Algebra in Quantum Theory, Encycl. Math., Vol.9, Addison-Wesley, Reading, Massachusetts 1981.

[12] R.S. Ingarden, A. Jamiolkowski, Classical Electrodynamics, Polish Sci. Publ. PWN, Warsaw 1985.

[13] J. Mozrzymas, Applications of Group Theory in Physics, Polish Sci. Publ. PWN, Warsaw 1976 (in Polish).

[14] T. Lulek, Acta Phys. Pol. A57, 407 (1980).

[15] B. Lulek, T. Lulek, Acta Phys. Pol. A66, 149 (1984).

[16] B. Lulek, T. Lulek, J. Phys. A, Math. Gen. 17, 3077 (1984).

[17] H. Weyl, The Theory of Groups and Quantum Mechanics, Menthuen, New York 1931.

[18] D.P. Zelobienko, Representation of Compact Lie Groups, Nauka, Moscow 1970 (in Russian). 